\title{
Instrumental piezoeléctrico comparado con sierra convencional en cirugía ortognática.
}

\section{Piezoelectric bone surgery compared to conventional saw in orthognathic surgery.}

\author{
Juan Pablo Vargas-Buratovic ${ }^{1,2}$, Catalina Paz López-Suárez ${ }^{1,2}$, Ana Sofía Rojas-Bascuñán ${ }^{1,2}$, Francisco Javier Pinedo-Henríquez ${ }^{1,2,3^{*}}$
}

\author{
1. Escuela de Odontología,Facultad de Medicina, \\ Pontificia Universidad Católica de Chile, Santiago, \\ Chile \\ 2. Proyecto Epistemonikos, Santiago, Chile \\ 3. Hospital Santiago Oriente - Dr. Luis Tisné \\ Brousse, Santiago, Chile. \\ * Correspondencia Autor: Francisco Pinedo \\ Dirección: Centro Evidencia UC, Pontificia \\ Universidad Católica de Chile, Diagonal \\ Paraguay 476, Santiago, Chile | E-mail: francisco. \\ pinedo@uc.cl
}

\begin{abstract}
RESUMEN
Introducción:La utilización de instrumental piezoeléctrico en cirugía ortognática ha ido en aumento con el fin de minimizar el riesgo de daño a tejidos blandos en comparación al uso de sierra convencional. Sin embargo, aún existe incertidumbre respecto a las complicaciones asociadas a cada instrumental. Métodos: Realizamos una búsqueda en Epistemonikos, la mayor base de datos de revisiones sistemáticas en salud, la cual es mantenida mediante el cribado de múltiples fuentes de información, incluyendo MEDLINE, EMBASE, Cochrane, entre otras. Extrajimos los datos desde las revisiones identificadas, analizamos los datos de los estudios primarios, realizamos un meta análisis y preparamos una tabla de resumen de los resultados utilizando el método GRADE. Resultados y conclusiones: Identificamos cuatro revisiones sistemáticas que en conjunto incluyeron 10 estudios primarios, de los cuales, cuatro corresponden a ensayos aleatorizados. Concluimos que el uso de instrumental piezoeléctrico en comparación con la sierra convencional podría disminuir el riesgo de daño nervioso grave y disminuir el sangrado grave $(>500$ $\mathrm{mL}$ ), pero la certeza de la evidencia es baja. Por otra parte, el uso de instrumental piezoeléctrico podría presentar poca o nula diferencia en el dolor postoperatorio, pero la certeza de la evidencia es baja. Finalmente no es posible establecer con claridad si el uso de instrumental piezoeléctrico disminuye la pérdida de sangre intraoperatoria (variable continua), la inflamación postoperatoria o el tiempo operatorio, ya que la certeza de la evidencia existente ha sido evaluada como muy baja.
\end{abstract}

\section{PALABRAS CLAVE}

Cirugía ortognática; Anomalía dentofacial; Instrumental piezoléctrico; Sierra convencional; Epistemonikos; GRADE.

\section{ABSTRACT}

Introduction: The use of piezoelectric bone surgery in orthognathic surgery has been increasing to minimize the risk of soft tissue damage compared to conventional saws. However, there is still uncertainty regarding the complications associated with each instrument. Methods: We searched in Epistemonikos, the largest database of systematic reviews in health, which is maintained by screening multiple information sources, including MEDLINE, EMBASE, Cochrane, among others. We extracted data from the systematic reviews, reanalyzed primary studies' data, conducted a metaanalysis and generated a summary of findings table using the GRADE approach. Results and conclusions: We identified four systematic reviews that included 10 primary studies, four of which corresponded to randomized trials. We conclude that the use of piezoelectric instrumentation compared to the conventional saw may reduce the risk of severe nerve damage and decrease severe bleeding $(>500 \mathrm{~mL})$, but the certainty of the evidence is low. On the other hand, the use of piezoelectric bone surgery may make little or no difference in postoperative pain, but the certainty of the evidence is low. Finally, we are uncertain whether piezoelectric bone surgery reduces intraoperative blood loss (continuous variable), postoperative inflammation, and operative time, as the certainty of the evidence has been assessed as very low.

\section{KEY WORDS}

Orthognatic surgery; Dentofacial Deformity; Piezoelectric bone surgery; Conventional Saw; Epistemonikos, GRADE.

Int. J. Inter. Dent Vol. 14(1); 73-78, 2021. 


\section{PROBLEMA}

La cirugía ortognática, es un procedimiento quirúrgico que tiene como objetivo corregir anomalías dentofaciales. Entre los pasos del procedimiento quirúrgico, se incluyen distintos tipos de osteotomías maxilares o mandibulares que tradicionalmente se han realizado por medio de instrumental rotatorio o sierras convencionales. Entre las desventajas de la utilización de este tipo de instrumental se ha descrito un daño accidental a tejidos blandos aumentando la incidencia de complicaciones intra o postoperatorias tales como daño a tejido nervioso o vasos sanguíneos ${ }^{(1)}$. Desde el año 2005 en adelante, el uso de instrumental piezoeléctrico en cirugía ortognática ha ido en aumento con el fin de minimizar el riesgo de daño a tejidos blandos y garantizar un campo quirúrgico menos contaminado ${ }^{(2,3)}$. Este sistema se basa en microvibraciones ultrasónicas que son transferidas a una punta de vibración (inserto) capaz de producir un fenómeno de cavitación sobre tejido duro como el hueso(2), y su frecuencia de vibración para la realización de osteotomías presenta un rango que fluctúa entre los 24 y $32 \mathrm{kHz}^{(1,2)}$. De forma diferente, la sierra convencional funciona en base a una ablación mecánica tras el corte y cizallamiento de la superficie ósea accionada por un micromotor, y su velocidad de acción depende del tamaño y rugosidad de la superficie dentada, junto a la velocidad de la pieza de mano y la presión ejercida sobre el hueso(18).

Pese a que se ha intentado demostrar la superioridad de un instrumental por sobre el otro, aún existe incertidumbre respecto a cuál intervención es la más efectiva y segura.

\section{MÉTODOS}

Realizamos una búsqueda en Epistemonikos, la mayor base de datos de revisiones sistemáticas en salud, la cual es mantenida mediante búsquedas en múltiples fuentes de información, incluyendo MEDLINE, EMBASE, Cochrane, entre otras. Extrajimos los datos desde las revisiones identificadas y analizamos los datos de los estudios primarios. Con esta información, generamos un resumen estructurado denominado FRISBEE (Friendly Summaries of Body of Evidence using Epistemonikos), siguiendo un formato preestablecido, que incluye mensajes clave, un resumen del conjunto de evidencia (presentado como matriz de evidencia en Epistemonikos), metanálisis del total de los estudios cuando sea posible, una tabla de resumen de resultados con el método GRADE y una sección de otras consideraciones para la toma de decisión.

\section{Mensajes clave}

- El uso de instrumental piezoeléctrico en comparación con la sierra convencional podría disminuir el daño nervioso grave a 1 o 2 meses y a los 6 meses, disminuir el sangrado grave y podría resultar en poca o nula diferencia en dolor postoperatorio (certeza de la evidencia baja).

- No es posible establecer con claridad si el uso de piezoeléctrico disminuye la pérdida de sangre intraoperatoria, el tiempo operatorio o la inflamación postoperatoria debido a que la certeza de la evidencia existente ha sido evaluada como muy baja.

- No se encontraron revisiones sistemáticas que evaluaran la calidad de vida posterior a la utilización de instrumental piezoeléctrico.

Acerca del conjunto de evidencia para esta pregunta

Encontramos cuatro revisiones sistemáticas ${ }^{(1,2,3,4)}$ que incluyeron 10 estudios primarios $(5,6,7,8,9,10,11,12,13,14)$ de los cuales cuatro corresponden a ensayos aleatorizados ${ }^{(8,9,12,14)}$.

Cuál es la evidencia

Véase matriz de evidencia en Epistemonikos más abajo.

Qué tipo de pacientes incluyeron los estudios*

Qué tipo de intervenciones incluyeron los estudios*

Qué tipo de desenlaces midieron
Un ensayo ${ }^{(8)}$ fue excluido por comparar el instrumental piezoeléctrico con instrumental rotatorio sólo en genioplastías.

Esta tabla y el resumen en general se basa en los ensayos restantes ${ }^{(9,12,14)}$ y en dos estudios observacionales ${ }^{(11,13)}$ que reportaban los desenlaces de interés y entregaban información adicional relevante para la toma de decisiones.

Todos los estudios incluyeron pacientes adultos entre 18 y 40 años ${ }^{(9,11,12,13,14)}$, con anomalías dentofaciales con indicación de cirugía ortognática.

Un estudio observacional ${ }^{(13)}$, y un ensayo $^{(14)}$ sólo incluyeron pacientes con anomalía dentofacial clase III.

Todos los estudios excluyeron pacientes con patologías sistémicas que contraindican en cirugía electiva. Además, un estudio observacional|(13) excluyó pacientes con historia de cirugía ortognática previa, trauma maxilofacial o cirugía de reconstrucción facial y un ensayo ${ }^{(14)}$ excluyó pacientes embarazadas o en etapa de lactancia.

Dos estudios observacionales ${ }^{(11,13)}$ y dos ensayos ${ }^{(12,14)}$ incluyeron osteotomías bimaxilares (osteotomía Le Fort I modificada y osteotomía sagital de rama mandibular) realizadas mediante el uso de instrumental piezoeléctrico o sierra convencional. Por otro lado, un ensayo ${ }^{(9)}$ solo incluyó osteotomía Le Fort I modificada.

Todos los procedimientos fueron realizados bajo anestesia general.

Los estudios reportaron múltiples desenlaces, los cuales fueron agrupados por las revisiones sistemáticas de la siguiente manera:

- Daño nervioso grave

- Sangrado grave (>500 mL)

- Pérdida de sangre intraoperatoria $(\mathrm{mL})$

- Tiempo operatorio (minutos)

- Dolor postoperatorio

- Inflamación postoperatoria

- Complicaciones intraoperatorias (fractura quirúrgica mandibular desfavorable y daño al nervio alveolar inferior)

El seguimiento de los estudios tuvo un rango que fluctuó entre 3 meses y 6 meses.

* La información sobre los estudios primarios es extraída desde las revisiones sistemáticas identificadas, no directamente desde los estudios, a menos que se especifique lo contrario. 


\section{RESUMEN DE LOS RESULTADOS}

La información sobre los efectos del uso de instrumental piezoeléctrico en cirugía ortognática está basada en un ensayo aleatorizado ${ }^{(14)}$ que incluyó 110 pacientes y en dos estudios observacionales ${ }^{(11,13)}$ que incluyeron 148 pacientes. El ensayo midió los desenlaces daño nervioso grave a 1 o 2 meses, daño nervioso grave a 6 meses y sangrado grave $(>500 \mathrm{~mL})$ (110 pacientes) $)^{(14)}$

Por otra parte, los desenlaces pérdida de sangre intraoperatoria y tiempo operatorio solo fueron evaluados por dos estudios observacionales que incluyeron 148 pacientes ${ }^{(11,13)}$

Ninguna revisión permitió la extracción de datos de los desenlaces "dolor" e "inflamación" de manera que pudieran ser incorporados a un metanálisis, por lo que la información de dichos desenlaces se presenta como síntesis narrativa.

El resumen de los resultados es el siguiente:

- El uso de instrumental piezoeléctrico en comparación con la sierra convencional podría disminuir el daño nervioso grave a 1 o 2 meses (baja certeza de la evidencia).

- El uso de instrumental piezoeléctrico en comparación con la sierra convencional podría disminuir el daño nervioso grave a los 6 meses (baja certeza de la evidencia).

- El uso de instrumental piezoeléctrico en comparación con la sierra convencional podría disminuir el sangrado grave $(>500 \mathrm{~mL}$ ) (baja certeza de la evidencia).

- No es posible establecer con claridad si el uso de piezoeléctrico disminuye la pérdida de sangre intraoperatoria debido a que la certeza de la evidencia existente ha sido evaluada como muy baja.

- No es posible establecer con claridad si el uso de piezoeléctrico disminuye el tiempo operatorio debido a que la certeza de la evidencia existente ha sido evaluada como muy baja.

- El uso de instrumental piezoeléctrico en comparación con la sierra convencional podría resultar en poca o nula diferencia en dolor postoperatorio (baja certeza de la evidencia).

- No es posible establecer con claridad si el uso de instrumental piezoeléctrico disminuye la inflamación postoperatoria debido a que la certeza de evidencia existente ha sido evaluada como muy baja.

- No se encontraron revisiones sistemáticas que evaluaran la calidad de vida de los pacientes sometidos a cirugía ortognática con instrumental piezoeléctrico en comparación con sierra convencional.

\section{Instrumental piezoeléctrico comparado con sierra convencional en cirugía ortognática}

\begin{tabular}{|c|c|c|c|c|}
\hline $\begin{array}{l}\text { Pacientes } \\
\text { Intervención } \\
\text { Comparación }\end{array}$ & \multicolumn{4}{|c|}{$\begin{array}{l}\text { Pacientes sometidos a cirugía ortognática } \\
\text { Instrumental piezoeléctrico } \\
\text { Sierra convencional }\end{array}$} \\
\hline \multirow{3}{*}{ Desenlaces } & \multicolumn{2}{|c|}{ Efecto absoluto* } & \multirow{3}{*}{$\begin{array}{l}\text { Efecto } \\
\text { relativo } \\
\text { (IC } 95 \%)\end{array}$} & \multirow{3}{*}{$\begin{array}{l}\text { Certeza de } \\
\text { la evidencia } \\
\text { (GRADE) }\end{array}$} \\
\hline & CON & $\begin{array}{l}\text { CON } \\
\text { instrumental } \\
\text { piezoeléctrico }\end{array}$ & & \\
\hline & \multicolumn{2}{|c|}{ Diferencia: pacientes por 1000} & & \\
\hline \multirow{2}{*}{$\begin{array}{l}\text { Daño nervioso } \\
\text { grave a } 102 \\
\text { meses }\end{array}$} & 509 por 1000 & 10 por 1000 & \multirow{2}{*}{$\begin{array}{l}\text { RR } 0,02 \\
(0,001 \mathrm{a} \\
0,28)\end{array}$} & \multirow{2}{*}{$\begin{array}{l}\oplus \oplus \bigcirc^{1,2} \\
\text { Baja }\end{array}$} \\
\hline & $\begin{array}{r}\text { Diferencia } \\
\text { (Margen de error: }\end{array}$ & $\begin{array}{l}99 \text { menos } \\
57 \text { a } 509 \text { menos) }\end{array}$ & & \\
\hline \multirow{2}{*}{$\begin{array}{l}\text { Daño nervioso } \\
\text { grave a los } 6 \\
\text { meses }\end{array}$} & 145 por 1000 & 9 por 1000 & \multirow{2}{*}{$\begin{array}{l}\text { RR } 0,06 \\
(0,001 \text { a } \\
0,99)\end{array}$} & \multirow{2}{*}{$\underset{\text { Baja }}{\oplus \bigcirc^{1,2}}$} \\
\hline & \multicolumn{2}{|c|}{$\begin{array}{l}\text { Diferencia: } 136 \text { menos } \\
\text { (Margen de error: } 1 \text { a } 145 \text { menos) }\end{array}$} & & \\
\hline \multirow{2}{*}{$\begin{array}{l}\text { Sangrado grave } \\
(>500 \mathrm{~mL})\end{array}$} & 527 por 1000 & 11 por 1000 & \multirow{2}{*}{$\begin{array}{l}\text { RR } 0,02 \\
(0,001 \mathrm{a} \\
0,27)\end{array}$} & \multirow{2}{*}{$\underset{\mathrm{Baja}}{\oplus \bigcirc^{1,2}}$} \\
\hline & \multicolumn{2}{|c|}{$\begin{array}{c}\text { Diferencia: } 517 \text { menos } \\
\text { (Margen de error: } 385 \text { a } 527 \text { menos) }\end{array}$} & & \\
\hline \multirow{2}{*}{$\begin{array}{l}\text { Pérdida de } \\
\text { sangre } \\
\text { intraoperatoria } \\
(\mathrm{mL})\end{array}$} & $311,7 \mathrm{~mL}$ & $162,76 \mathrm{~mL}$ & \multirow[b]{2}{*}{--} & \multirow[b]{2}{*}{$\begin{array}{l}\bigoplus \bigcirc \bigcirc O^{3,4,5} \\
\text { Muy Baja }\end{array}$} \\
\hline & \multicolumn{2}{|c|}{$\begin{array}{c}\text { DM: } 148,94 \mathrm{~mL} \text { menos } \\
\text { (Margen de error: } 303,57 \mathrm{~mL} \text { menos a } 5,7 \mathrm{~mL} \\
\text { más) }\end{array}$} & & \\
\hline \multirow{2}{*}{$\begin{array}{l}\text { Tiempo } \\
\text { operatorio }\end{array}$} & 238 minutos & 214,54 minutos & \multirow[b]{2}{*}{--} & \multirow{2}{*}{$\begin{array}{l}\oplus \bigcirc \bigcirc \bigcirc^{3,4,5} \\
\text { Muy Baja }\end{array}$} \\
\hline & \multicolumn{2}{|c|}{$\begin{array}{l}\text { DM: } 23,46 \text { menos } \\
\text { (Margen de error: } 44,48 \text { menos a } 91,4 \text { más) }\end{array}$} & & \\
\hline $\begin{array}{l}\text { Dolor } \\
\text { postoperatorio }\end{array}$ & \multicolumn{3}{|c|}{$\begin{array}{c}\text { Una revisión sistemática [1] que se basó en un ensayo (30 } \\
\text { participantes) [9] reportó que no existieron diferencias } \\
\text { entre los grupos al evaluar el dolor postoperatorio medido } \\
\text { al segundo y tercer día. }\end{array}$} & $\underset{\mathrm{Baja}}{\oplus \bigcirc^{1,2}}$ \\
\hline
\end{tabular}




\begin{tabular}{|c|c|c|c|}
\hline \multirow{2}{*}{$\begin{array}{l}\text { Inflamación } \\
\text { postoperatoria }\end{array}$} & \multicolumn{2}{|c|}{$\begin{array}{c}\text { Una revisión sistemática [1] que se basó en un estudio } \\
\text { observacional ( } 12 \text { participantes) [13] reportó una mayor } \\
\text { inflamación postoperatoria en los procedimientos } \\
\text { realizados con sierra convencional en comparación con el } \\
\text { instrumental piezoeléctrico. }\end{array}$} & \multirow{2}{*}{$\begin{array}{c}\oplus \bigcirc \bigcirc \bigcirc 2,3 \\
\text { Muy Baja } \\
\text {-- }\end{array}$} \\
\hline & & -- & \\
\hline \multicolumn{4}{|c|}{ 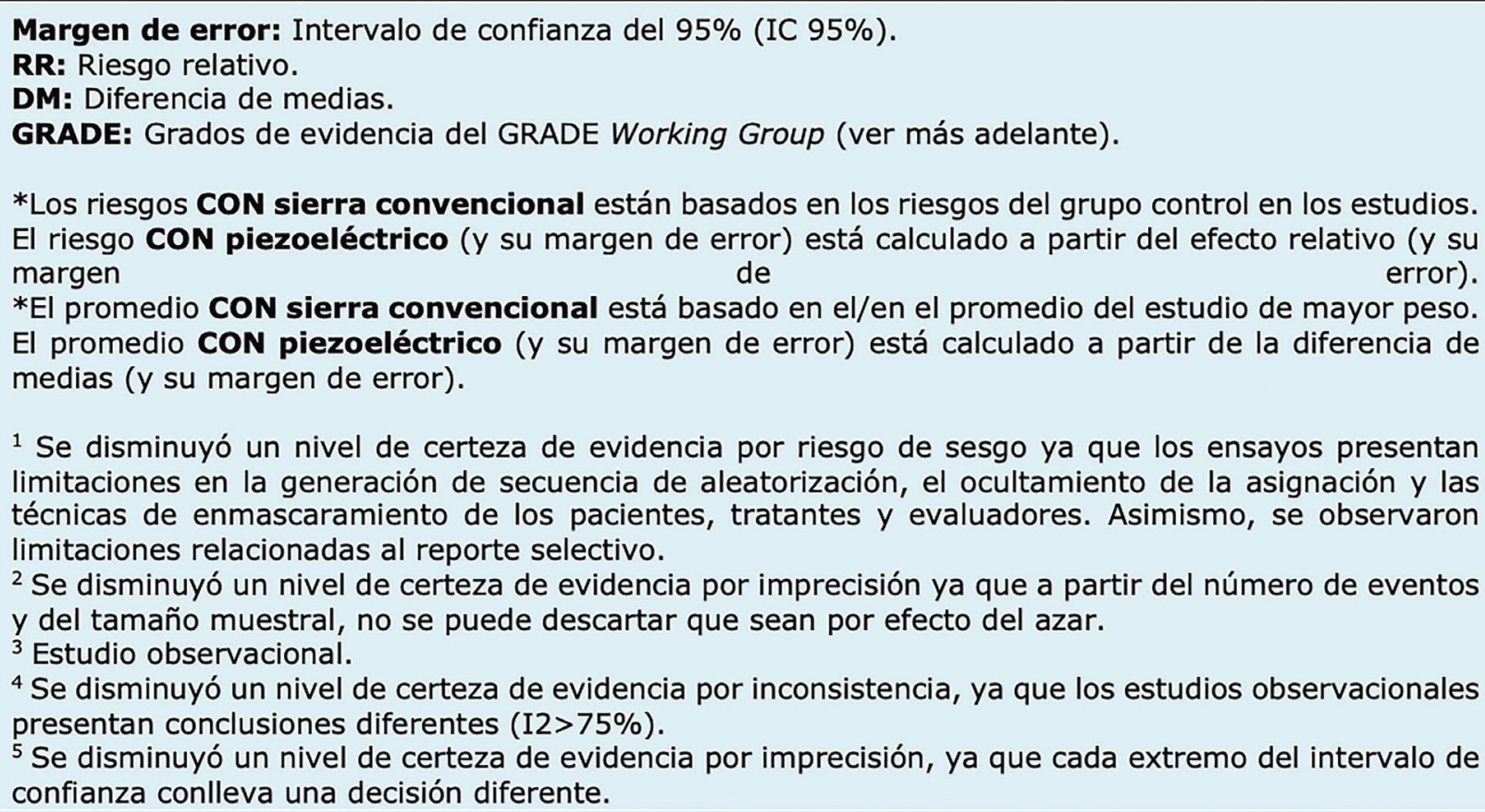 } \\
\hline
\end{tabular}

\section{Acerca de la certeza de la evidencia (GRADE)*}

$\bigoplus \oplus \oplus \oplus$

Alta: La investigación entrega una muy buena indicación del efecto probable. La probabilidad de que el efecto sea sustancialmente distinto ${ }^{+}$es baja.

\section{$\oplus \oplus \oplus \bigcirc$}

Moderada: La investigación entrega una buena indicación del efecto probable. La probabilidad de que el efecto sea sustancialmente distinto ${ }^{\dagger}$ es moderada.

\section{$\oplus \oplus \bigcirc \bigcirc$}

Baja: La investigación entrega alguna indicación del efecto probable. Sin embargo, la probabilidad de que el efecto sea sustancialmente distinto ${ }^{\dagger}$ es alta.

\section{$\oplus \bigcirc 0 \mathrm{O}$}

Muy baja: La investigación no entrega una estimación confiable del efecto probable. La probabilidad de que el efecto sea sustancialmente distinto ${ }^{\dagger}$ es muy alta.

*Esto es también denominado 'calidad de la evidencia' o 'confianza en los estimadores del efecto'. tSustancialmente distinto = una diferencia suficientemente grande como para afectar la decisión 


\section{OTRAS CONSIDERACIONES PARA LA TOMA DE DECISIÓN}

\section{A quién se aplica y a quién no se aplica esta evidencia}

- Los resultados de este resumen son aplicables a pacientes adultos con anomalías dentofaciales tratadas mediante cirugía ortognática bimaxilar.

- Esta evidencia no aplica a pacientes con patologías sistémicas no controladas, ni a pacientes con antecedentes de cirugía ortognática previa, trauma maxilofacial o reconstrucción facial.

- Por otra parte existen contraindicaciones relativas sobre el uso de instrumental piezoeléctrico en pacientes portadores de un desfibrilador cardioversor implantable (DCl), por la posibilidad de interferencia electromagnética y alteración en el funcionamiento del dispositivo. Sin embargo, no existe consenso sobre esta posible contraindicación ya que existe evidencia que muestra que no se observan alteraciones en el funcionamiento del $\mathrm{DCl}$ al utilizar instrumental piezoeléctrico en la cavidad oral ${ }^{(15)}$

\section{Sobre los desenlaces incluidos en este resumen}

- Los desenlaces incluidos en la tabla de resumen de hallazgos son aquellos considerados críticos para la toma de decisiones de acuerdo a la opinión de los autores de este resumen y se encuentran en general en concordancia con las revisiones sistemáticas identificadas.

- El desenlace calidad de vida del paciente posterior a ser sometido a la intervención no fue reportado por ninguna revisión sistemática.

\section{Balance daño/beneficio y certeza de la evidencia}

- El conjunto de evidencia muestra un posible beneficio en la disminución de daño nervioso grave a 1 o 2 meses, daño nervioso grave a los 6 meses y sangrado grave $(>500 \mathrm{~mL})$ al utilizar instrumental piezoeléctrico en comparación a la sierra convencional en cirugía ortognática bimaxilar.

- Por otro lado, el uso de instrumental piezoeléctrico podría no presentar diferencias en el dolor postoperatorio en comparación con la sierra convencional, pero la certeza de evidencia es baja.

- Si bien se observa una disminución en la pérdida de sangre intraoperatoria, el tiempo operatorio y la inflamación postoperatoria, existe considerable incertidumbre ya que la certeza de la evidencia es muy baja para estos desenlaces.

- A partir de lo anterior, la decisión pudiese estar a favor del uso de instrumental piezoeléctrico por sobre instrumental convencional, pero debido a la incertidumbre de la evidencia existente no es posible realizar un adecuado balance riesgo/beneficio.

\section{Consideraciones de recursos}

- No es posible determinar con exactitud la costo-efectividad de la intervención con instrumental piezoeléctrico, pero se ha reportado un mayor costo en la adquisición del instrumental piezoeléctrico ${ }^{(16)}$. Por otra parte, este tipo de análisis no fue reportado por ninguna revisión sistemática.

- Por otra parte, entendiendo al tiempo de utilización de pabellones quirúrgicos como un recurso limitado, se requiere la publicación de ensayos clínicos que evalúen el tiempo operatorio asociado a cada instrumental. Si bien basados en los estudios observacionales disponibles, obtuvimos una diferencia de medias a favor del uso de instrumental piezoeléctrico, la certeza de la evidencia fue evaluada como muy baja, lo cual impide obtener resultados concluyentes al comparar ambas intervenciones.

\section{Qué piensan los pacientes y sus tratantes}

- Si bien los resultados obtenidos en este resumen podrían favorecer el uso de instrumental piezoeléctrico en cirugía ortognática, debe considerarse la disponibilidad de este instrumental junto a las preferencias y experiencia del cirujano tratante.

- Los tratantes podrían tener una preferencia por el uso de instrumental piezoeléctrico debido a que requiere una menor necesidad de protección de tejidos blandos y la obtención de un campo quirúrgico más limpio, producto de la disminución del sangrado intraoperatorio y la continua irrigación por parte del mismo instrumental. Esto podría traducirse en un menor riesgo de hipovolemia postquirúrgica y su consecuente transfusión sanguínea. No obstante, la necesidad de transfusión sanguínea post cirugía ortognática es extremadamente baja ${ }^{(2)}$

- Por otro lado, basados en la evidencia disponible, los pacientes también podrían preferir el uso de instrumental piezoeléctrico debido a la posible disminución del sangrado y daño nervioso. De todas formas se requiere de nuevos estudios que evalúen los resultados reportados por los pacientes (PROs), para describir sus percepciones respecto a las intervenciones a las que sean sometidos.

\section{Diferencias entre este resumen y otras fuentes}

- Las conclusiones de este resumen coinciden en general con las de las revisiones sistemáticas identificadas respecto a la disminución en el daño nervioso, sangrado grave, pérdida de sangre intraoperatoria, dolor postoperatorio, inflamación postoperatoria y tiempo operatorio al utilizar instrumental piezoeléctrico comparado con sierra convencional ${ }^{(1,2,3,4)}$

- No se identificaron guías de práctica clínica que evalúen el uso de instrumental piezoeléctrico ni de sierra convencional en cirugía ortognática.

\section{¿Puede que cambie esta información en el futuro?}

- La probabilidad de que una investigación futura cambie las conclusiones de este resumen es alta, considerando la incertidumbre de la evidencia existente.

- Luego de realizar una búsqueda en International Clinical Trials Registry Platform de la Organización Mundial de la Salud no se identificaron registros de ensayos clínicos en curso.

- Por otro lado, en la base de datos PROSPERO se identificó una revisión sistemática ${ }^{(16)}$ que abordaría la pregunta de interés.

\section{CÓMO REALIZAMOS ESTE RESUMEN}

Mediante métodos automatizados y colaborativos recopilamos toda la evidencia relevante para la pregunta de interés y la presentamos en una matriz de evidencia.

Siga el enlace para acceder a la versión interactiva: Instrumental piezoeléctrico comparado con sierra convencional en cirugía ortognática.

\section{NOTAS}

Si con posterioridad a la publicación de este resumen se publican nuevas revisiones sistemáticas sobre este tema, en la parte superior de la matriz se mostrará un aviso de "nueva evidencia".

Este artículo es parte del proyecto síntesis de evidencia de Epistemonikos. Se elabora con una metodología preestablecida, siguiendo rigurosos estándares metodológicos y proceso de revisión por pares interno. Cada uno de estos artículos corresponde a un resumen, denominado FRISBEE (Friendly Summary of Body of Evidence using Epistemonikos), cuyo principal objetivo es sintetizar el conjunto de evidencia de una pregunta específica, en un formato amigable a los profesionales clínicos. Sus principales recursos se basan en la matriz de evidencia de Epistemonikos y análisis de resultados usando metodología GRADE. Mayores detalles de los métodos para elaborar este FRISBEE están descritos aquí (http://dx.doi.org/10.5867/medwave.2014.06.5997)

La Fundación Epistemonikos es una organización que busca acercar la información a quienes toman decisiones en salud, mediante el uso de tecnologías. Su principal desarrollo es la base de datos Epistemonikos (www.epistemonikos.org).

\section{DECLARACIÓN DE CONFLICTOS DE INTERESES}

Los autores declaran no tener conflictos de intereses con la materia de este artículo.

\section{AGRADECIMIENTOS}

Este resumen de evidencia fue elaborado con el apoyo metodológico del Centro Evidencia UC, Facultad de Medicina, Pontificia Universidad Católica de Chile. 


\section{Bibliografía}

1. Rude K, Svensson P, Starch-Jensen T. Neurosensory disturbances after bilateral sagittal split osteotomy using piezoelectric surgery: a systematic review. J Ora Maxillofac Surg. 2019 Feb;77(2):380-90.

2. Pagotto LEC, de Santana Santos T, de Vasconcellos SJA, Santos JS, MartinsFilho PRS. Piezoelectric versus conventional techniques for orthognathic surgery: Sys-tematic review and meta-analysis. J Craniomaxillofac Surg. 2017 Oct:45(10):1607-13.

3. AlAsseri N, Swennen G. Minimally invasive orthognathic surgery: a systematic re-view. Int J Oral Maxillofac Surg. 2018 Oct;47(10):1299-310.

4. Thereza-Bussolaro C, Galván Galván J, Pachêco-Pereira C, Flores-Mir C. Maxillary osteotomy complications in piezoelectric surgery compared to conventional surgi-cal techniques: a systematic review. Int J Oral Maxillofac Surg. 2019 Jun;48(6):720-31.

5. Beziat JL, Bera JC, Lavandier B, Gleizal A. Ultrasonic osteotomy as a new tech-nique in craniomaxillofacial surgery. Int J Oral Maxillofac Surg. 2007 Jun;36(6):493-500.

6. Shirota T, Kamatani T, Yamaguchi T, Ogura H, Maki K, Shintani S. Effectiveness of piezoelectric surgery in reducing surgical complications after bilateral sagittal split osteotomy. Br J Oral Maxillofac Surg. 2014 Mar;52(3):219-22.

7. Monnazzi MS, Real Gabrielli MF, Passeri LA, Cabrini Gabrielli MA, Spin-Neto R, Pereira-Filho VA. Inferior alveolar nerve function after sagittal split osteotomy by reciprocating saw or piezosurgery instrument: prospective double-blinded study. J Oral Maxillofac Surg. 2014 Jun;72(6):1168-72.

8. Rullo R, Festa VM, Rullo F, Trosino O, Cerone V, Gasparro R, et al. The use of pie-zosurgery in genioplasty. J Craniofac Surg. 2016 Mar;27(2):414-5

9. Rana M, Gellrich NC, Rana M, Piffkó J, Kater W. Evaluation of surgically assisted rapid maxillary expansion with piezosurgery versus oscillating saw and chisel osteotomy - a randomized prospective trial. Trials. 2013 Feb 17;14:49.

10. Landes CA, Stübinger S, Rieger J, Williger B, Ha TK, Sader R. Critical evaluation of piezoelectric osteotomy in orthognathic surgery: operative technique, blood loss, time requirement, nerve and vessel integrity. J Oral Maxillofac Surg. 2008 Apr;66(4):657-74.

11. Landes CA, Stübinger S, Ballon A, Sader R. Piezoosteotomy in orthognathic surgery versus conventional saw and chisel osteotomy. Oral Maxillofac Surg. 2008 Sep;12(3):139-47.

12. Brockmeyer P, Hahn W, Fenge S, Moser N, Schliephake H, Gruber RM Reduced somatosensory impairment by piezosurgery during orthognathic surgery of the mandible. Oral Maxillofac Surg. 2015 Sep;19(3):301-7.

13. Spinelli G, Lazzeri D, Conti M, Agostini T, Mannelli G. Comparison of piezosurgery and traditional saw in bimaxillary orthognathic surgery. J Craniomaxillofac Surg. 2014 Oct;42(7):1211-20.

14. Bertossi D, Lucchese A, Albanese M, Turra M, Faccioni F, Nocini $P$, et al. Piezosur-gery versus conventional osteotomy in orthognathic surgery: a paradigm shift in treatment. J Craniofac Surg. 2013;24(5):1763-6.

15. Gómez G, Jara F, Sánchez B, Roig M, Ferrer R, Duran-Sindreu F. Safety concerns of piezoelectric units in implantable cardioverter defibrillator. J Oral Maxillofac Surg. 2018 Feb;76(2):273-7.

16. Yaman Z, Suer BT. Piezoelectric surgery in oral and maxillofacial surgery. Annals of Oral \& Maxillofacial Surgery. 2013; 1(1):5

17. Salah M, Hakam M, Radi IA, Abdel-Aziz O. Post-surgical neurosensory dysfunction of inferior alveolar nerve in bilateral sagittal spilt osteotomy of the mandible using saw versus piezotome: a systematic review and meta-analysis. PROSPERO 2016 CRD42016037974 Available from: https://www.crd.york.ac.uk/ prospero/display record.php?ID=CRD42016037974

18. Troedhan A, Mahmoud ZT, Wainwright M, Khamis MM. Cutting bone with drills burs, lasers and piezotomes: A comprehensive systematic review and recommendations for the clinician. Int J Oral Craniofac Sci. 2017;3(2):020-033. 